\title{
ILCEA
}

Revue de l'Institut des langues et cultures

d'Europe, Amérique, Afrique, Asie et Australie

16 | 2012

La culture progressiste à l'époque de la guerre froide

\section{Abstraktion: Weltsprache oder Ausdruck der „dekadenten westlichen Moderne"?}

L'abstraction, langage universel ou expression du « modernisme occidental décadent »?

\section{Patrice Neau}

\section{OpenEdition}

\section{Journals}

Édition électronique

URL : http://journals.openedition.org/ilcea/1489

DOI : 10.4000/ilcea.1489

ISSN : 2101-0609

Éditeur

UGA Éditions/Université Grenoble Alpes

Édition imprimée

ISBN : 978-2-84310-232-5

ISSN : $1639-6073$

Référence électronique

Patrice Neau, „Abstraktion: Weltsprache oder Ausdruck der "dekadenten westlichen Moderne"?", ILCEA

[Online], 16 | 2012, Online erschienen am: 04 Juli 2012, abgerufen am 22 März 2021. URL: http:// journals.openedition.org/ilcea/1489; DOI: https://doi.org/10.4000/ilcea.1489

Ce document a été généré automatiquement le 22 mars 2021.

(C) ILCEA 


\title{
Abstraktion: Weltsprache oder Ausdruck der „dekadenten westlichen Moderne“?
}

\author{
L'abstraction, langage universel ou expression du « modernisme occidental \\ décadent »?
}

Patrice Neau

1 Schon vor der Gründung der beiden deutschen Staaten 1949 entbrannte ein Kunststreit zwischen Ost und West. Nach den zwölf Jahren der NS-Herrschaft stellte sich die Frage der ästhetischen und kunstwissenschaftlichen Verortung der bildenden Künste in Deutschland. Anders als nach der Niederlage von 1918, wo ein revanchistischer Diskurs entwickelt worden war, war das Thema der "deutschen Kunst“ in der Nachkriegszeit ein Tabu (Belting, 1992). Wichtiger war es in den ersten Nachkriegsjahren, den Anschluss an die internationalen Entwicklungen seit 1933 zu ermöglichen. Die Moderne des 20. Jahrhunderts, die Werke der „entarteten“ Künstler sollten rehabilitiert werden. „Befreite Kunst“ (so der Name einer Ausstellung in Celle 1946) wurde in Ost und West in vielen Ausstellungen, die von den Kulturoffizieren der Besatzungsmächte organisiert worden waren, einem kulturhungrigen Publikum präsentiert. ${ }^{2}$

Die Debatten um die bildenden Künste können nicht von dem politischen und ideologischen Hintergrund des Kalten Krieges und der Teilung Deutschlands abgekoppelt werden. Dass sie sich in den 50er Jahren um die Abstraktion, die von Werner Haftmann zur Weltsprache erhoben wurde, kristallisieren werden, war von vornherein nicht vorauszusehen. Der konservative Widerstand gegen moderne Kunst (Abstraktion, aber auch Expressionismus und Surrealismus) hatte sich schon Ende der 40er Jahre gebildet: Sedlmayrs Anklage gegen die Moderne, Verlust der Mitte, ${ }^{3}$ bildete die ideologische Begründung des Antimodernismus. In der SBZ wurde nach einer kurzen Phase der Rehabilitierung die Moderne bewusst und zielstrebig diffamiert: Ziel der Angriffe durch die Presse der SBZ waren vor allem der Expressionismus und die 
Abstraktion. In Ost und West wusste man, welche Bedeutung der Kunst als Erziehungs(oder Umerziehungsmittel) beizumessen war.

3 Wie kam es dazu, dass die Abstraktion im Westen kanonisiert wurde, dass sie in die ästhetische Kontinuität der so genannten „klassischen Moderne“ gestellt wurde und sozusagen als Inbegriff der Freiheit und der Westintegration der Bundesrepublik dargestellt wurde, während sie im Osten als antihumanistisch, volksfremd und dekadent verschrien wurde?

\section{Die Allgemeine Deutsche Kunstausstellung Dresden}

4 Die Jahre vor dem Beginn des Kalten Krieges schienen von der Möglichkeit eines kulturellen Dialogs zwischen Ost und West zu zeugen. Als Beweis dafür wird immer wieder auf die „Allgemeine Deutsche Kunstausstellung“ in Dresden (25.08. bis 29.10.1946) hingewiesen. Der Kunsthistoriker Will Grohmann und der Maler Hans Grundig waren in die französische und in die amerikanische Zone ausgesandt worden, um bei Künstlern und Galeristen nach Werken von namhaften Malern und Bildhauern $\mathrm{zu}$ suchen. Tatsächlich wurden in Dresden unter den 600 Exponaten Werke der wichtigsten deutschen Künstler ausgestellt: Ernst Ludwig Kirchner, Oskar Kokoschka, Otto Dix, Max Beckmann, Ernst Barlach, Willi Baumeister, Ernst Wilhelm Nay, George Grosz, Oskar Schlemmer, Karl Hofer, Karl Schmidt-Rotluff, Otto Müller unter anderen. Moderne aber Epigonentum waren aber wahllos, so konnte es scheinen, nebeneinander ausgestellt. Die Ausstellung stellte, so Bernd Lindner „eine nur bedingt gelungene Mischung aus bedeutenden Werken der Moderne vor 1933 und vielen Arbeiten dar, die weniger kraft ihres künstlerischen Ranges als aufgrund ihres dokumentarischen Charakters oder aus Mangel an besseren Objekten ausgestellt worden waren [...]“ (Lindner, 1998, S. 77). Eine Ausstellung solchen Ausmaßes im zerstörten Dresden ein Jahr nach Beendigung des Krieges konnte natürlich nicht ohne die Einwilligung und die tatkräftige Unterstützung der SMAD stattfinden. Sie konnte vordergründig als Zeichen der kulturellen Offenheit der SBZ gedeutet werden, sie war aber Teil einer geschickten Propagandakampagne. In einer Ruinenlandschaft zeigte die SBZ ihre Überlegenheit: nur in Dresden konnte eine umfassende Ausstellung organisiert werden, nur die SBZ konnte die materiellen Hindernisse überwinden, um allen Deutschen in West und Ost die Kunstentwicklungen zu zeigen, die ihnen zwölf Jahre lang vorenthalten worden waren. Die sehr eklektische Auswahl sollte ein Massenpublikum anziehen, es wurde gezielt um die „bürgerliche Intelligenz“ geworben, an den Geschmack eines konventionellen Bildungsbürgertums angeknüpft, was den Zielen der von Stalin verordneten Bündnispolitik entsprach.

Die Dresdner Ausstellung wurde aber von Anfang an von der SED instrumentalisiert. Ihr Sprachrohr, die Sächsische Zeitung berichtete ausführlich, aber nur scheinbar sachlich, über den Verlauf der Ausstellung: bejahende, aber auch immer mehr kritische Stimmen wurden laut (Lindner, 1998, S. 71-74). Gegner und Verteidiger der Ausstellung konnten sich zu Worte melden, so dass ein Streit um die „rechte Kunst“ entbrannte und Fronten sich abzuzeichnen begannen: einerseits die Verfechter der Moderne, die in der Minderheit waren, und andererseits eine unselige Allianz aus „Ewiggestrigen“, die über die „entarteten Künstler“ ungehindert herziehen konnten und Parteiideologen, die sich nun auf eine durch eine Umfrage bestätigte „Stimme der Besucher“ berufen konnten. Anlässlich dieser Ausstellung war eine Umfrage unter den Besuchern durchgeführt 
worden. Das Ergebnis dieser Untersuchung zeigte, wie tief sich der Wortschatz des Nazismus in die Köpfe eingeprägt hatte: $15 \%$ der Befragten lehnte Werke ab, weil sie von „Geisteskranken“ gemalt worden seien, 8\% weil sie „entartet“ seien (Lindner, 1998, S. 70-81). ${ }^{4}$ Vor allem an der expressionistischen und an der abstrakten Kunst wurde Kritik geübt, obwohl die Maler, die diese Kunstrichtungen vertraten, zahlenmäßig in der Minderheit waren. Die Umfrage wurde von der kurz vorher gegründeten SED benutzt, um eine volksnahe, ästhetisch konventionelle, realistische, volksverbundene, anspruchslose und sich an den Alten Meistern orientierende Kunst zu propagieren, auch wenn von einer generellen Ablehnung der Moderne durch das Publikum nicht gesprochen werden konnte. ${ }^{5}$ Sorgfältig ausgewählte Besuchermeinungen sollten dann die Legimitierung für die spätere Kulturpolitik der SED liefern. Will Grohmann ist selber Opfer dieser Instrumentalisierung gewesen, indem er als bekannter Kunsthistoriker und ausgewiesener Kenner der Moderne in die westlichen Zonen geschickt wurde, um die Ausstellung mit Werken beschicken zu können, die dann den Zorn der Besucher erregen würden.

6 Schon einige Monate vor der Eröffnung der Allgemeinen Ausstellung in Dresden hatte sich Anton Ackermann ${ }^{6}$ unmissverständlich geäußert: wenn er Freiheit für Wissenschaft und Kunst versprach, so machten Einschränkungen, in einer Sprache, die ihre Nähe zur LTI kaum verhehlen konnte, die Hoffnungen der Künstler zunichte, die auf eine Autonomie der Kunst hätten pochen wollen: „Wenn dann aber irgendein Pseudokünstler herkommt, um Zoten über den Humanismus, die Freiheit und die Demokratie [...] zu reißen, dann soll er das ,gesunde Volksempfinden' genauso spüren wie der Pseudowissenschaftler." (Gillen, 2005, S. 31.)

71946 sind die Weichen schon gestellt. In dieser Hinsicht erfüllte die Dresdner Ausstellung eine Doppelfunktion:

- nach außen hin, für die breite Öffentlichkeit in Ost und West, wurde der Wille der deutschen Kulturorgane der SBZ bekundet, sich als weltoffen und liberal zu zeigen und für ein geeinigtes Deutschland zu plädieren. So konnte auf die „Intelligenz“ zugegangen werden. Den Künstlern wurde zeitweilig ein Spielraum gewährt, der nach dem Ende der „Bündnispolitik“ zurückgenommen wurde;

- für den parteiinternen Gebrauch wurde die Stimmung der „gesunden Volksmassen“ so dahin interpretiert, dass moderne, fremde Kunstrichtungen in der SBZ als unerwünscht erschienen. Der Fragebogen, der sorgfältig ausgewertet wurde, wurde für die späteren kulturpolitischen Pläne der SED genutzt: schon 1946 findet man die Argumente, die dann in dem Formalismusstreit benutzt wurden.

\section{Der Formalismusstreit}

Gegen den Anspruch der SED und der ihr untergeordneten Organisationen, dem Kulturleben strenge Parteirichtlinien aufzuzwingen, regte sich aber Widerspruch. Der Konflikt wurde in der Zeitschrift bildende kunst öffentlich ausgetragen. Der Architekt Adolf Behne, Mitherausgeber der Zeitschrift mit Oskar Nerlinger, versuchte mit Karl Hofer, der selber später heftig gegen die Abstraktion polemisieren sollte, die Moderne gegen die Wortführer des sozialistischen Realismus in Schutz zu nehmen. ${ }^{8}$ Auch wenn schon 1946 Oberst Tulpanow und Major Dymschitz in Reden (anlässlich der Dresdner Ausstellung und des Allgemeinen Sächsischen Künstlerkongresses. z. B.) versucht hatten, die Künstler auf die von Shdanow propagierten Linie des sozialistischen 
Realismus einzuschwören, bildete der Artikel von Dymschitz in der Täglichen Rundschau „Über die formalistische Richtung in der deutschen Malerei“9 den Auftakt zu einem Generalangriff gegen alle renitenten Künstler.

Auch im Bereich der Kultur hatte die Politik der ersten Nachkriegsjahre unter dem Zeichen der Blockpolitik gestanden: die Zusammensetzung der gegründeten Kulturorganisationen spiegelte diese von der SED verfolgte Strategie wider: Vertreter aller Kunstrichtungen wurden in die Vorstände dieser Gremien gewählt, die SED aber stellte den Vorsitzenden und besetzte die strategischen Stellen. Der Anschein eines gewissen Liberalismus wurde bis 1948 aufrechterhalten: die Parteifunktionäre, unterstützt von den sowjetischen Kulturoffizieren, propagierten zwar mit sehr mäßigem Erfolg eine Ausrichtung auf den sozialistischen Realismus, während die Mehrzahl der bildenden Künstler diese ideologische und ästhetische Bevormundung ablehnte. Da viele in der SBZ schaffende Künstler im III. Reich als entartete Künstler abgestempelt worden waren, durften sich abweichende Meinungen noch äußern.

Der Beginn des Kalten Krieges führte zur Verschärfung des ideologischen Kampfes, da die Sowjetunion auf einen Konfrontationskurs mit dem Westen zu steuerte. Die erfolglose Berliner Blockade und die Währungsreform im Juni 1948 beschleunigten den Prozess der Spaltung. In diesem neuen politischen Kontext konnte Stalin nicht mehr an dem Traum eines ungeteilten, neutralen Deutschland unter alliierter Oberaufsicht festhalten.

11 Im Kalten Krieg wurde Kunst als ideologische Waffe von beiden Seiten eingesetzt. Nicht zufällig 1948 wurde in mehreren deutschen Städten die Wanderausstellung "Gegenstandslose Malerei aus Amerika“ gezeigt, die Bilder aus der Guggenheim Foundation zeigte, deren Direktorin die deutsche Künstlerin Hilla von Rebay war. Dass die CIA in den 50er Jahren das Zustandekommen von Kunstausstellungen unterstützt hat, ist kein Geheimnis mehr. Hier offenbarte sich wahrscheinlich nur ein Manko der amerikanischen Politik: auswärtige Kulturpolitik wurde in den USA bis zu diesem Zeitpunkt klein geschrieben.

12 Zur gleichen Zeit entstand in New York das spätere Guggenheim-Museum: „es handelt sich um die Annäherung modernen Lebensgefühls und ungegenständlicher Malerei, die in ihrem neuen Heim harmonisch verbunden werden sollen" notierte Egon Vietta in der Zeit. ${ }^{10}$ Dieses neue Lebensgefühl, das sich hier äußerte, war schon die Ahnung der kommenden Wohlstandsgesellschaft der 50er Jahre und die Etablierung der „Weltsprache Abstraktion“ in der Bundesrepublik kann von den gesellschaftlichen Trends der Nachkriegszeit nicht getrennt werden: Abstraktion lag im Zeitgeist, war Bestandteil eines Lebensgefühls. Gegen den sich schon abzeichnenden Siegeszug der Abstraktion als westdeutsche "Geltungskunst" (Rehberg) hatte die SBZ schon eine ideologische Mauer errichtet, den sozialistischen Realismus.

Die Formalismuskampagne wurde 1951 neu aufgerollt, das Ziel des sozialistischen Realismus wurde in der Formel des „Neuen Menschen“ umgeschrieben. Lenins Maxime, die Parteilichkeit und Volkstümlichkeit als wichtigste Kriterien der Kunst definierte, wurde in die Praxis umgesetzt. Die dritte Deutsche Kunstausstellung in Dresden im März 1953 fand, nach dem ideologischen Scheitern der zweiten Aufstellung 1949, unter dem Motto des „Durchbruchs zum sozialistischen Realismus“ statt.

14 Kann die Entwicklung der westdeutschen Malerei in den fünfziger Jahren als Antwort auf die starre Doktrin des sozialistischen Realismus, auf eine vereinnahmte Kunst 
gedeutet werden? Kann sie aber auch von der vorangegangenen Kunstpolitik des nationalsozialistischen Deutschland verstanden werden? Konnte an die Moderne der Weimarer Republik (Kandinsky, Klee) angeknüpft werden, als sei nichts geschehen? Oder sollten neue Wege gesucht werden? Die Debatte erhielt 1950 eine neue Dimension und gab der gegenstandslosen Kunst den entscheidenden Impuls. Diesen Impuls verdankte sie ironischerweise auch dem katholisch-konservativen Kunsthistoriker Hans Sedlmayr: seine maßlosen Angriffe gegen die gesamte Moderne, seine Behauptung, „die moderne Kunst sei ein Symptom für eine allgemeine Entartung "11 diskreditierten jede restaurative Tendenz. Willi Baumeister, Johannes Itten, aber auch Alexander Mitscherlich und Theodor W. Adorno verteidigten die Abstraktion als zeitgemäßen Ausdruck der Zeit.

Im Rückblick auf die fünfziger Jahre gilt das „Erste Darmstädter Gespräch“ heute als Auslöser einer Bewegung, in deren Verlauf sich die westdeutsche Kunstszene von der gegenständlichen Darstellung mehr und mehr verabschiedet hat. Zugleich erfuhr die abstrakte Kunst in dieser Veranstaltung ihre erste öffentlich vollzogene spirituelle Auratisierung auf deutschem Boden nach dem Zweiten Weltkrieg, die sich nachfolgend auf den beiden ersten documenta-Veranstaltungen für viele Jahre verfestigen sollte. ${ }^{12}$

\section{Die documenta}

Die erste documenta (1955) präsentierte sich als „internationale Ausstellung“, sie wies aber noch eine nationale Orientierung auf (nur sechs Nationen waren vertreten). Sie diente der „Vergangenheitsbewältigung“ in dreifacher Hinsicht (Kimpel, 1997):

- Deutschland musste über die gesamte künstlerische Entwicklung seit 1933, seit der Abkoppelung von den anderen Ländern informiert werden (die westlichen Alliierten hatten aber auf Zonenebene schon Vorarbeit geleistet). Sie war als Gegenausstellung zu den „Großen deutschen Kunstsausstellungen“ des III. Reichs zu verstehen, aber auch zu den deutschen Kunstausstellungen der DDR in Dresden.

- Sie war als Zeichen für die Wiedergutmachung im moralischen Sinne gedacht: die Künstler, deren Werke zerstört oder verkauft worden waren, sollten rehabilitiert werden. 58 deutsche Künstler wurden gezeigt, davon 31, die im Dritten Reich als „entartet“ galten. Die Ausstellung wies aber Lücken auf: der deutsche Expressionismus wurde honoriert, der italienische Futurismus sowie der Konstruktivismus waren gut vertreten, die realistische Kunst wurde aber ausgeblendet (Grasskamp sprach von einer ,wohlwollenden Fälschung der Moderne "). ${ }^{13}$ Werner Haftmann hatte soeben seine Malerei im XX. Jahrhundert. Eine Entwicklungsgeschichte, in deren Mittelpunkt die Behauptung von der Abstraktion als einziger Weltsprache steht, veröffentlicht.

- Die documenta 1 galt auch einer gesellschaftspolitischen „Standortbestimmung“. Zehn Jahre nach der Kapitulation war die Bundesrepublik politisch, wirtschaftlich, militärisch im Westen fest verankert, sie war in der so genannten Freien Welt angekommen. Die Standortbestimmung diente als Brücke zwischen Vergangenheit und Zukunft und entsprach den kulturpolitischen Interessen der jungen Bundesrepublik, auch wenn die politische Aufgabe nicht explizit als Zielvorgabe formuliert wurde: der freie Westen zeigte seine Leistungsfähigkeit auch auf dem Boden der bildenden Künste, wenige Kilometer vom eisernen Vorhang entfernt. Diese Ausstellung war keine staatlich inszenierte Kunstausstellung wie in der DDR, sie entsprang einer privaten Initiative, einem Verein, der den unglücklichen Namen trug: „Abendländische Kunst des XX. Jahrhunderts e. V.“ Schon 
der Name lässt aufhorchen: er klingt nicht nur bieder-konservativ, provinziell, er hat im Deutschland der 50er Jahre eine sehr klare Konnotation. Es ist die Bekenntnisvokabel zu der politischen Tradition des christlichen Abendlandes, das gegen den antichristlichen Bolschewismus verteidigt werden muss. Krieges. Sie hat sich international behaupten können, denn sie war mehr als eine bloße Kunstausstellung, sie besa $\beta$ fast eine theoretische Dimension und wirkte präskriptiv auf Museen, Kuratoren, Sammler, Kritiker und auf den Kunstmarkt. Sie bestimmte den gültigen, herrschenden Kunstbegriff. Sie stellte nicht nur eine Diagnose über den Zeitgeist, sie bezog auch Stellung, indem sie die Abstraktion zur Norm erhob.

\section{Abstraktion als Weltsprache}

17 Werner Haftmann lieferte die ideologische Begründung der Alleingültigkeit der Abstraktion, deren Gegner nicht „die ehrlichen Traditionalisten, die unentwegt Völkischen, die warnenden Humanisten, usw“ (Haftmann, 1954, S. 421) seien, sondern alles, was Haftmann unter dem Begriff Totalitarismus subsumierte:

Der Totalitarismus ist der Oberbegriff, unter dem scheinbar so gegensätzliche Bewegungen wie der Bolschewismus der leninistisch-stalinistischen Phase, der Faschismus Mussolinis und der Nationalsozialismus Hitlers in enge Nachbarschaft geraten. Als verblüffendsten und deutlichsten Ausdruck dieser inneren, gegen die menschliche Freiheit gerichteten Übereinstimmung hat er exakt die gleiche Kunstanschauung hervorgebracht. Der offizielle Kunststil totalitärer Länder ist überall der gleiche.

Es begann in Russland. ${ }^{14}$

Im Osten hatte der sozialistische Realismus gesiegt, im Westen wurde die Abstraktion als einzig zeitgemäße ästhetische Ausdrucksform legitimiert. Abstraktion als Chiffre der Moderne integrierte die Bundesrepublik in die Denkstrukturen der freien Welt. Der Rückblick über die Kunst seit der Jahrhundertwende anlässlich der documenta 1 sollte eine kathartische Wirkung haben: er reinigte Deutschland von den Verbrechen des III. Reiches, einer Zeit, die aber ausgeklammert wurde. Gleichzeitig aber wurde der restaurative Charakter der Adenauer-Ära durch Kreation überwunden, ohne Fragen an die Geschichte zu stellen. Rückblickend hat sich Günter Grass in seiner Rede zum 8. Mai sehr kritisch zu dieser Allgegenwart der Abstraktion geäußert:

Es ging auch um das Wahrnehmen oder Übersehen der Wirklichkeit in einem Land, das geschlagen, geteilt war, dessen zu verantwortende Last Völkermord hie $\beta$ und das dennoch oder deshalb im Begriff war, alles zu verdrängen, ich sage, gegenstandslos zu machen, was die Vergangenheit heraufbeschwören und die Flucht nach vorn behindern konnte. ${ }^{15}$

Die d 2 (1959) verstand sich in der Folge der documenta 1 als Apotheose der Abstraktion: der Weg, der mit Cézanne anfing und der über den Kubismus zur Abstraktion führte, verurteilte alle anderen Kunstformen zur Bedeutungslosigkeit. Wenn die dl die Abstraktion offensiv betrieb und sie gegen ihre konservativen Widersacher verteidigen musste, bedurfte die documenta 2 keiner Verteidigungsstrategie mehr. Sie belegte nur den Sieg der Abstraktion. Haftmann erklärte, die Abstraktion entwerfe das Bild einer „künstlerischen Gemeinschaft von wahrhaft globalem Ausmaß. Ihre Sprache, die Sprache der modernen Kunst wird in der ganzen Welt gesprochen und verstanden“ (Kimpel, 1997, S. 260). So wurde die 
Abstraktion zu einer anthropologischen Wahrheit erhoben. Haftmann war es gelungen, die abstrakte Kunst konsensfähig und $\mathrm{zu}$ einem Erlebnis für breite Schichten $\mathrm{zu}$ machen. Abstraktion war modern, sie entsprach dem Zeitgeist, sie verdrängte, ob sie es wollte oder nicht, die unerwünschten Schatten der jüngsten Vergangenheit. Haftmanns Blick blieb aber europazentriert und er integrierte Deutschland in die Gemeinschaft der europäischen Völker, deren kulturelle Überlegenheit in einem fast imperialistischen Duktus gefeiert wurde:

Von Europa bis Amerika, von Kanada bis Brasilien, von Persien bis Japan hat [der europäische Lebensentwurf] die oft über Jahrtausende aufgebauten und gehaltenen Kulturbastionen der Folklore überwältigt und an der Oberfläche vernichtet. An ihm beginnt die Weltkultur sichtbar zu werden. ${ }^{16}$

Damit signalisierte Haftmann Kontinuität. Die Kunst, die im III. Reich und auch schon vor 1933 von einem konservativen Publikum geächtet worden war, war jetzt rehabilitiert - oder totgeschwiegen: von kritischen Künstlern wie George Grosz oder John Heartfield ${ }^{17}$ war jetzt in der documenta nicht mehr die Rede. Dieser „neue Idealismus" (so Belting) ersetzte die Gräuel der geschehenen Geschichte durch entpolitisierte Bilder: „In diesem Geschichtsverständnis lag der Impuls aus der Gegenwart, welche denn auch von anderen, in den USA nämlich, künstlerisch entdeckt wurde." ${ }^{18}$ Vielleicht weil sie als von allen gesellschaftlichen und historischen Bindungen befreit erschien, wurde die abstrakte Kunst als das Symbol einer freien Gesellschaft von der Öffentlichkeit und von den Multiplikatoren überraschend schnell akzeptiert.

Die amerikanischen Künstler waren bei der documenta 2 gut vertreten (10\% der Werke, und vor allem Großformate). Eine Ausstellung, die sachlich und informativ sein wollte, konnte nicht anderes als den amerikanischen abstrakten Expressionismus zeigen. Für die Amerikaner war die documenta 2 ein willkommener Anlass, den weltweiten Erfolg der amerikanischen Kunst zur Geltung zu bringen und den Anschluss an die Kunstgeschichte zu finden: die amerikanische Kunst, die bis 1945 ein unbeschriebenes Blatt in Europa gewesen war, fand jetzt Eingang in die Kunstgeschichte.

Der Erfolg der Abstraktion kann aber nur unzureichend mit der geballten Kraft der amerikanischen Beeinflussungspolitik erklärt werden. Der Beginn einer allmählichen aktiven Unterstützung der abstrakten Maler kann bis zu der Zeit der Dresdner Kunstausstellung 1946 zurückverfolgt werden, zeigt aber, dass die Vereinigten Staaten sehr pragmatisch, ohne vorher fest gefassten Plan vorgegangen sind. Ende 1946 galt es, den autoritären Prämissen der ostdeutschen Kulturpolitik entgegenzuwirken und die Unabhängigkeit der Kunst von der politischen Führung zu schützen. Indem die in Dresden angegriffene Kunst nun unterstützt wurde, konnte die politische Unabhängigkeit der Kunst als wirksames Symbol der individuellen Freiheit verkündet werden, was zum Paradoxon führte, dass die Kunst dadurch ihre Autonomie einbüßte.

Man darf die Frage aufwerfen, ob der Dresdner Angriff von 1946 nicht zu einer zuerst nicht intendierten Förderung der Abstraktion geführt hat. Abstraktion war nun die Antwort auf die Kulturpolitik der Ostzone und bot den Amerikanern die Möglichkeit, die "Westkunst" scharf von dem sozialistischen Realismus abzuheben. Dass die Abstraktion zu diesem Zeitpunkt in Amerika - nicht zuletzt durch die Vermittlung ausgewanderter deutscher Künstler - erfolgreich war, ist eine andere Geschichte. Dass sie dann offiziell unterstützt wurde, ist nicht die Folge einer auswärtigen Kulturpolitik, die es noch nicht gab, sondern vielmehr das Ergebnis einer pragmatischen Politik, 
dessen doppelter Nutzen offensichtlich wurde: so konnte der Osten kulturell bekämpft werden, so konnte auch endlich die amerikanische bildende Kunst, die in Europa noch unrezensiert war, einem breiten Publikum zugänglich gemacht werden. Diese tatkräftige Unterstützung der Abstraktion entspringt mehr der Eigendynamik des Abgrenzungsprozesses zwischen den Blöcken als einer zielbewussten Kulturpolitik.

\section{Die Abstraktion und die DDR}

Nicht nur die Abstraktion, sondern alle Richtungen der Moderne waren im Zug der Formalismuskampagne verurteilt worden und mussten der verordneten optimistischen Darstellung einer idyllischen sozialistischen Gesellschaft weichen. Autonome Strömungen existierten zwar weiter, wurden aber der Öffentlichkeit entzogen.

In der DDR-internen Diskussion wurde die documenta unreflektiert verteufelt. In völliger Unkenntnis der ersten documenta schrieb der Kunstkritiker Walter Hänel: „Die erste documenta war bereits ein Zeugnis ausgesprochener Kulturbarbarei, erfüllt von Pessimismus und Zerstörung." ${ }^{\text {"19 }}$ Ihre Initiatoren, Bode und Haftmann wurden als Theoretiker einer Verfallskunst gebrandmarkt, die documenta als Bestandteil des „Machtmechanismus der herrschenden Monopolkreise“ verurteilt. Die „abstraktionistische Kunstdiktatur“ (so Hänel) wurde für die DDR-Kulturfunktionäre zum Werk der westdeutschen Monopole erklärt, die den Markt beeinflusst und manipuliert hätten, um das Entstehen einer gesellschaftskritischen Kunst zu verhindern:

Die abstrakte Kunst und ihre Theoretiker leisten einen aktiven Beitrag zur Bonner Staatspolitik, zum Antikommunismus, indem sie auf der einen Seite das sozialistische Kunstschaffen in der Sowjetunion, der DDR und den übrigen sozialistischen Ländern gegenüber der westdeutschen Künstlerschaft verleumden und jegliche realistische Kunst in Westdeutschland zu unterdrücken versuchen. Auf der anderen Seite sind sie bestrebt, den dekadenten westlichen Modernismus als fünfte Kolonne in die sozialistischen Länder einzuschleusen, um auf die künstlerische Intelligenz dieser Länder zersetzend einzuwirken. ${ }^{20}$

Mit dem „bürgerlichen“ Kunsthistoriker Werner Haftmann wurde nach dem Erfolg der ersten documenta hart ins Gericht gegangen. Da die marxistische Kunstgeschichte Kunst als Form der Wirklichkeitserkenntnis betrachtete und sie auch so auslegte, dass in den Kunstwerken die Wirklichkeit (allerdings die erstrebte Wirklichkeit einer idealen, noch nicht verwirklichten sozialistischen Gesellschaft) dargestellt werden sollte, wurde die Abstraktion als Ausdruck einer bürgerlich-dekadenten Anschauung verurteilt. Den Malern der Abstraktion wurde vorgeworfen, das nationale und kulturelle Erbe zu zerstören und durch ihr Wirken nicht den gesellschaftlichen Aufgabenstellungen der neuen Gesellschaft Rechnung zu tragen. Das so genannte „spätbürgerliche Kunsterbe“, das im Westen nun rehabilitiert worden war, sollte, weil geschichtspessimistisch und elitär, überwunden werden. Dabei wurde immer wieder auf die internationale Situation hingewiesen: die Debatte um die Verortung der sozialistischen Kunst ist Teil der ideologischen Auseinandersetzung zwischen den beiden gesellschaftlichen Systemen. Deshalb genügte es nicht, den Bildenden Künstlern in der DDR durch Zwangsmaßnahmen die Richtung vorzuschreiben, der Kampf gegen die Abstraktion musste eine ideologische Legitimation im Kampf gegen die "psychologische Kriegführung“ des „Bonner Revanchismus“ erhalten. 

der Bundesrepublik gespielt hat, kann nicht geleugnet werden. Insbesondere der im August 1951 gegründete „Kulturkreis der deutschen Wirtschaft im BDI“ spielte eine zentrale Rolle in der Förderung und Verbreitung der zeitgenössischen Kunst. Der Kulturkreis bemühte sich durch Spenden und Dauerleihgaben um die Unterstützung der Museen beim Aufbau ihrer Sammlungen. Da sich die Kunst der Nachkriegszeit der Abstraktion verschrieben hatte, wurde sie von dem Kulturkreis gefördert. Für Hänel handelte es sich aber um eine bewusste Strategie der Monopole, um Künstler zu fördern, die im Dienste einer Ideologie standen und dadurch gleichzeitig durch Stipendien, Ankäufe von Kunstwerken, Ausstellungen das Kunstleben indirekt zu lenken und realistische Künstler ihres Lebensunterhalts $\mathrm{zu}$ berauben und auszugrenzen. Hänel übersieht die Komplexität des Vorganges, der tatsächlich zur vorübergehenden Vorherrschaft der Abstraktion geführt hat. Dass der BDI mit seiner Unterstützung der modernen Kunstrichtungen eine politische Strategie verfolgte, ist nicht von der Hand zu weisen. Der Kulturkreis, als Teil der zivilen Gesellschaft, unterstützte das Kunstschaffen, die Kreation. Durch seine Tätigkeit (die jährlich stattfindende Ausstellung ars viva z. B.) wirkte er präskriptiv, indem er junge, noch unbekannte Nachwuchskünstler förderte.

30

Was Hänel außer acht ließ, ist die Tatsache, dass das zeitweilige Vorherrschen einer Kunstrichtung nicht von einer „unsichtbaren Hand“ gelenkt wurde: er übertrug hier nur das autoritäre Modell der DDR, wo eine bestimmt nicht unsichtbare eiserne Hand das Kunstschaffen lenkte. Das komplexe und immer etwas mysteriöse Zusammenwirken von Museumsdirektoren, Kuratoren, Galeristen, Kunstkritikern, Sammlern wurde von Hänel völlig ignoriert. Er übersah auch, dass der Erfolg der Abstraktion in den 50er Jahren in die gesellschaftlichen Trends der westdeutschen Nachkriegsgesellschaft eingebettet war. Sie bildete eine Zeitlang den Mainstream. Ihre Anziehungskraft war die der im Entstehen begriffenen Konsumgesellschaft. Der Erfolg der Abstraktion war unzertrennlich von der Ausbreitung einer Massengesellschaft. Die moderne Kunst vermittelte ein neues Lebensgefühl, sie formte das Gesicht einer neuen, Optimismus und Wohlstand verströmenden Welt: „Modern art in your life“ hie $\beta 1949$ eine Ausstellung im New Yorker MOMA, deren Grundidee 1952 in Recklinghausen wieder aufgegriffen wurde (,Mensch und Form unserer Zeit") und wo versucht wurde „durch erlesene Werke der bildenden Kunst und der Literatur, durch technische Geräte, Möbel und Hausrat die Form unserer Zeit sichtbar zu machen.“" ${ }^{22}$ Wie Laszlo Glozer schrieb: „Die Nutzung der Kunst unterhalb dessen, was sie an Problem und Widerspruch in sich trägt, gelang damals prächtig.“" ${ }^{23}$ 


\section{Schlussbemerkungen} 50er Jahre auf mehrere Faktoren gründet.

- Dieser Erfolg war von dem Wunsch getragen, sich von der Vormundschaft der Ideologien zu befreien: dies erklärt die vehemente Ablehnung der Thesen von Sedlmayr, aber auch die Vorbehalte gegen einen Realismus, der Gefahr laufen könnte, mit dem sozialistischen Realismus gleichgesetzt zu werden. Abstraktion wurde somit mit Freiheit gleich gesetzt, und Freiheit als Gegenpol zum real existierenden Sozialismus war das Motto dieser Jahre.

- Er kann auch als Ausdruck der Verdrängung einer Auseinandersetzung mit der unmittelbaren Vergangenheit verstanden werden und als Rückzug in eine Kunst ohne subversive Kraft, die zwar das Leiden, den Verlust ausdrücken kann, ohne aber Fragen nach der gesellschaftlichen und historischen Verantwortung zu stellen.

- Der unwiderstehliche Sog der Moderne umfasst nicht nur die bildenden Künste, sondern alle Aspekte des „modern way of life“. Die westdeutsche Gesellschaft sucht eine Verankerung in der Gegenwart, in einer Gesellschaft, die von dem Konsum des Wirtschaftswunders geprägt ist und für die Amerika, in fast jeder Hinsicht, das Vorbild bildet. Die „konsumgerechte Westkunst“" (Eduard Beaucamp) verkam zu einem Massenphänomen, sie war Bestandteil einer optimistischen Konsumgesellschaft geworden

BeLting Hans, Das Ende der Kunstgeschichte. Eine Revision nach zehn Jahren, München, Verlag

C. H. Beck, 1995.

-, Die Deutschen und ihre Kunst. Ein schwieriges Erbe, München, Verlag C. H. Beck, 1992.

GILLEN Eckhart, Das Kunstkombinat DDR. Zäsuren einer gescheiterten Kunstpolitik, Köln, DuMont, 2005.

GLOZER Laszlo, Westkunst. Zeitgenössische Kunst seit 1939, DuMont, Köln, 1981.

HAFTMANN Werner, Malerei im 20. Jahrhundert, München, Prestel-Verlag, 1954.

HÄNEL Walter, Maler, Mäzene, Monopole, Berlin, Dietz Verlag, 1967.

KIMPEL Harald, documenta. Die Überschau, Köln, DuMont, 2002.

-, documenta, Mythos und Wirklichkeit, Köln, DuMont, 1997.

LINDNER Bernd, Verstellter, offener Brief. Eine Rezeptionsgeschichte bildender Kunst im Osten Deutschlands 1949-1995, Köln, Weimar, Wien, Böhlau, 1998.

REHBERG Karl-Siegbert und KAISER Paul (Hrsg.), Abstraktion im Staatssozialismus. Feindsetzungen und Freiräume im Kunstsystem der DDR, Weimar, VDG, 2003.

SEDLMAYR Hans,Verlust der Mitte. Die bildende Kunst des 19. und 20. Jahrhunderts als Symptom und Symbol der Zeit, Salzburg, Otto Müller, 1948. 
THOMAs Karin, Kunst in Deutschland seit 1945, Köln, DuMont, 2002.

\section{NOTES}

1. C'est la thèse que développe Werner Haftmann dans son histoire de l'art du $\mathrm{xx}^{\mathrm{e}}$ siècle : Malerei im 20. Jahrhundert. Eine Entwicklungsgeschichte, Munich, 1954.

2. Im Juni 1946 fand in Konstanz eine umfassende Ausstellung moderner Kunst: „Neue deutsche Kunst“, in Augsburg eröffnete die amerikanische Militärregierung eine Ausstellungsreihe mit dem Titel „Maler der Gegenwart“, in Baden-Baden, Freiburg und natürlich auch Berlin wurden umfassende Ausstellungen organisiert.

3. Der Kunsthistoriker Hans Sedlmayr war von 1936 bis 1945 an der Universität Wien tätig, ab 1951 in München: Sein polemisches Werk Verlust der Mitte erschien 1948.

4. Id., ibid., S. 70-81.

5. Nur 84 Fragebögen sind erhalten geblieben. Es waren vor allem die abstrakten Bilder, die auf Ablehnung stießen. Lindner hebt hervor, dass es aber falsch wäre, „von einer Ablehnung der Moderne schlechthin“ (Hervorhebung im Text) zu sprechen. Siehe dazu B. Lindner, Verstellter, offener Blick, S. 79.

6. Anton Ackermann (1905-1973) war der Leiter einer der drei Gruppen deutscher Kommunisten, die schon am 30. April 1945 nach Berlin, Sachsen und Mecklenburg-Pommern eingeflogen wurden. Er vertrat bis 1948 die in der SED weit verbreitete Hoffnung auf einen eigenen deutschen Weg zum Sozialismus.

7. LTI: Lingua Tertii Imperii. So bezeichnet der Romanist Victor Klemperer die Sprache des Dritten Reiches. Siehe dazu: V. Klemperer, LTI - Notizbuch eines Philologen, Berlin, 1947.

8. A. Behne, „Was will die moderne Kunst?“, in bildende kunst, 1/1948; K. Hofer, „Kunst und Politik“, ibid., 10/1948. Beide verteidigen die Freiheit der Kunst gegen Oskar Nerlinger, der die Notwendigkeit der Volksverbundenheit der Kunst betonte.

9. Tägliche Rundschau, 24.11.1948. Die Tägliche Rundschau war das Organ der SMAD.

10. „Gegenstandslose Malerei. Eine amerikanische Schau in Karlsruhe“, Die Zeit, Nr. 15, 15. April 1948, S. 5.

11. Zitiert in K. Thomas, Kunst in Deutschland seit 1945, Köln, DuMont, 2002, S. 47.

12. Ibid., S. 47.

13. Zitiert in Harald Kimpel, documenta. Die Überschau, Köln, DuMont, 2002, S. 22.

14. W. Haftmann, Malerei im 20. Jahrhundert, München, Prestel-Verlag, 1954, S. 421.

15. G. Grass, „Geschenkte Freiheit. Rede zum 8. Mai 1945“, in G. Grass, Essays, Reden, Briefe, Kommentare, Luchterhand, Darmstadt/Neuwied, 1987, S. 899.

16. W. Haftmann, Malerei im 20. Jahrhundert, S. 479.

17. John Heartfield kehrte 1950 in die DDR zurück. Das Exil in England wurde ihm vorgehalten, sein Gesuch, in die SED aufgenommen zu werden, abgelehnt. Eine im Oktober 1950 geplante Ausstellung wurde wegen „Formalismus“ abgesagt. Georg Grosz, der 1933 in die USA emigriert war, fand dort keine künstlerische Anerkennung. Erst 1959 kehrte er nach Deutschland zurück, wo er wenig später verstarb.

18. H. Belting, Das Ende der Kunstgeschichte. Eine Revision nach zehn Jahren, München, Beck, 1995, S. 47

19. W. Hänel, Maler, Mäzene, Monopole, S. 155.

20. W. Hänel, Maler, Mäzene, Monopole, S. 183.

21. So zum Beispiel bei Walter Hänel, Maler, Mäzene, Monopole, Berlin, Dietz Verlag, 1967, S. 189.

22. Im Katalog der Ausstellung „Mensch und Form unserer Zeit“, Recklinghausen, 1952 zitiert in Laszlo Glozer, Westkunst. Zeitgenössische Kunst seit 1939, Köln, DuMont, 1981, S. 174. 
23. Ibid., S. 174.

\section{RÉSUMÉS}

Die Abstraktion, die Werner Haftmann anlässlich der zweiten documenta zur Weltsprache erhob, hat für etwa ein Jahrzehnt die Kunstszene der Bundesrepublik beherrscht und die gegenständliche Malerei ins Abseits gedrängt.

Der Erfolg der abstrakten Kunst in Westdeutschland wird mit dem Sieg einer neuen, internationalen Sprache identifiziert, welche die Autonomie des Künstlers betont. Die kulturpolitische Bedeutung der Abstraktion, ihre Anerkennung in den Medien, ihre Förderung durch die Institutionen und nicht zuletzt ihr universeller Anspruch setzen sie schon Ende der vierziger Jahre den Angriffen der sowjetischen Kulturoffiziere und dann der Kulturfunktionäre der DDR aus.

Der politischen, wirtschaftlichen und militärischen Eingliederung der Bundesrepublik in den westlichen Block entspricht also auch eine ästhetische Integration in die freie Welt, die westdeutsche Kunst wird nach einer Ausgrenzung von zwölf Jahren in die Gemeinschaft der freien Völker wieder einbezogen und grenzt sich somit sowohl von der nationalsozialistischen Vergangenheit als auch von der totalitären Staaten des Ostens ab.

Wenn im Westen die Autonomie der Kunst proklamiert wird, so sollen in der DDR die Künstler einen nationalen Auftrag erfüllen, und werden aufgefordert, den Anweisungen der Kulturfunktionäre und Parteiideologen zu folgen.

Dieser Beitrag setzt sich mit der Instrumentalisierung der abstrakten Kunst jenseits und diesseits des „Eisernen Vorhangs“ auseinander. Mehr als der kunsttheoretische und ästhetische Diskurs wird ein ideologisches Konstrukt hinterfragt, das im Osten die abstrakte Kunst als antihumanistisch, volksfeindlich und dekadent, als Instrument der Monopole und des BDI, als Negation des „klassischen Erbes“ verdammt, und im Westen die Kunst in eine ästhetische Kontinuität, die der „klassischen Moderne“, und in den Dienst der Versöhnung und der Westintegration stellt.

L'abstraction, proclamée lors de la seconde documenta langage universel par Werner Haftmann, a régné de manière absolue sur l'art de l'Allemagne de l'Ouest pendant une bonne dizaine d'années, reléguant la peinture figurative dans un provincialisme poussiéreux.

On identifie souvent le succès de l'art abstrait au triomphe d'un nouveau langage international, voire mondial qui va de pair avec l'affirmation de l'autonomie de l'artiste. L'omniprésence de l'art abstrait sur la scène artistique de l'Allemagne de l'Ouest, son succès médiatique, le soutien que lui apportent les institutions en font dès la fin des années 1940 la cible privilégiée de l'offensive que mènent les officiers culturels soviétiques, relayés par les fonctionnaires culturels de la jeune RDA contre toutes les formes d'expression artistique qui refusent de se plier au canon du réalisme socialiste.

À l'intégration politique, militaire et économique de la République fédérale dans le camp occidental correspond aussi une intégration esthétique qui replacerait, après ce qui ne serait plus qu'une «parenthèse" de douze ans, les arts plastiques allemand dans le mouvement ininterrompu de l'art moderne occidental ${ }^{1}$.

À cette volonté de légitimation idéologique qui prétend ériger l'autonomie de l'artiste en valeur suprême s'oppose à l'Est une politique culturelle qui elle aussi cherche à légitimer un système 
politique en convoquant les artistes, dorénavant auxiliaires du pouvoir politique, investis d'une mission nationale et à ce titre sommés de se plier à des préceptes dont ils ne peuvent s'éloigner qu'à la marge.

Nous étudierons donc comment l'art abstrait a, de part et d'autre du rideau de fer, fait l'objet d'une instrumentalisation dont le but est d'opposer, dans une dichotomie réductrice, deux systèmes de société. Nous ne nous attacherons pas tant aux discours théoriques et écrits esthétiques produits dans les deux Allemagnes qu'aux discours idéologiques qui d'un côté condamnent l'art abstrait, considéré comme l'expression artistique d'une société décadente et un instrument d'aliénation des masses aux mains des « grands monopoles capitalistes » et de l'autre l'utilisent pour replacer la République fédérale dans une continuité esthétique et l'ancrer dans un système de valeurs à portée universelle.

INDEX

Schlüsselwörter : Abstraktion, documenta, Formalismus, Werner Haftmann

Mots-clés : abstraction, documenta, formalisme, Werner Haftmann

\section{AUTEUR}

\section{PATRICE NEAU}

Université de Nantes 